\title{
Critical Care Medicine: Principle of Diagnosis and Management in the Adult
}

\author{
Joseph E. Parrillo, R. Phillip Dellinger (eds). Elsevier, Amsterdam, 2019, 5th edition, \\ Hardcover, ISBN 9780323446761
}

\author{
Ben Fultz, MD (i)
}

Received: 2 February 2019/Revised: 23 June 2019/Accepted: 1 July 2019/Published online: 24 July 2019

(C) Canadian Anesthesiologists' Society 2019

Critical Care Medicine: Principle of Diagnosis and Management in the Adult, 5th Edition lies somewhere between a desk reference and a practical review of critical care medicine. In a rapidly expanding and evolving field such as critical care, it is difficult to produce a balanced and complete - yet accessible and digestible - resource, but this book does it admirably. It is well-positioned in a field of contemporary intensive care texts e.g. Oh's Intensive Care Manual 8th Edition (A.D. Bersten and J.M. Handy, Editors), Comprehensive Critical Care: Adult 2nd Edition (P.R. Roberts, S.R. Todd, Editors), and Irwin \& Rippe's Intensive Care Medicine 8th Edition (R.S. Irwin, C.M. Lilly, P.H. Mayo, and J.M. Rippe, Eds). The two editors of this book are from New Jersey. Dr. Joseph E. Parillo is board certified in Internal Medicine, Allergy and Immunology, and Cardiovascular Disease, and Dr. R. Phillip Dellinger is certified in Internal Medicine, Pulmonary Disease, and Critical Care Medicine.

This 5th edition that addresses critical care in adults comprises 1,322 pages and is divided into nine sections authored by multiple international specialists in the field, although most are from the United States. The book starts with its longest section - Critical Care Procedures, Monitoring, and Pharmacology - which covers an assortment of critical care-related topics, including cardiac arrest and pacing, airway and ventilator management, hemodynamics and monitoring devices, bedside ultrasonography and echocardiography, renal replacement therapy, and procedures such as chest tube insertion, intracranial pressure monitoring, and tracheostomy. The subsequent parts of the book delve

B. Fultz, MD $(\bowtie)$

University of Manitoba, Winnipeg, MB, Canada

e-mail: fultz.ben@gmail.com deeper into specific topics related to cardiopulmonary, renal, neurologic, gastrointestinal, and hematologic systems; infectious disease; physical and toxic injuries (e.g., poisonings, burns); and administrative, ethical, and psychological aspects of critical care. Of note, there is not a section devoted specifically to organ donation.

The chapters in this text usually begin with a historical perspective of the topic at hand, providing a brief, yet descriptive, medical history behind the monitors, procedures, and therapies. This history serves as an interesting introduction that eases the reader into the subject. Often there is a concise epidemiologic overview, and the consensus definitions pertinent to the subject are clearly explained to anchor the reader's understanding. The pertinent body of evidence is reviewed from a broad perspective, with an appropriate review of landmark studies often accompanied by reproduced figures. The text is well referenced and provides sufficient detail to leave the reader with a consensus-based interpretation of the literature while acknowledging alternate viewpoints. Overall, the style from one section to the next in this text is quite consistent and there is minimal repetition, although within each chapter there is some content variability depending on the author (e.g., providing information about what is common practice at the writer's institution). Relevant guidelines are provided and explored in detail. Overall, the chapters are well organized and flow logically, building the reader's subject knowledge.

A commendable feature of this 5th Edition of Critical Care Medicine: Principle of Diagnosis and Management in the Adult is its figures. The text is replete with clear, highly colored, well-organized figures, tables, and flowcharts both real-life and schematic illustrations. Importantly, the figures are well integrated with the text, making the information highly accessible to the reader - even to those 
more inclined toward visual learning. Moreover, the text is supplemented with online-accessible videos (particularly the echocardiography and ultrasonography sections) to further facilitate visual understanding. Compared with contemporary competing critical care tomes (mentioned above), this book is distinguished by its figures, graphics, and online material, thus making it especially appealing and memorable.

Several chapters of the text are particularly well done. The chapters regarding percutaneous mechanical circulatory support and heart failure are well rounded and have numerous figures, diagrams, and real-life device images that make the physiologically challenging concepts (e.g., Impella H-Q curves, ECMO hemodynamics, impact of LV venting), much easier to understand. The chapter covering acute respiratory distress syndrome provides an excellent physiologic review that considers the various prognostic factors as well as a pragmatic bedside approach to managing the ventilator. The chapter on renal replacement therapy contains an excellent, concise summary of the various trials that have addressed the dosing, timing, and initiation of renal replacement therapy in critically ill patients. The echocardiography chapter is very strong, with helpful photographs and easily accessible online video content. The chapter entitled Effect of Critical Illness on Pharmacokinetics and Pharmacodynamics is highly informative, considering drug dosing alterations needed in the presence of obesity, pregnancy, and/or renal or hepatic failure. Finally, the chapters on infectious diseases are noteworthy. Within fewer than 100 pages, the authors break down a vast topic into a manageable assortment of tables and figures without losing important details. There are helpful figures detailing (among other subjects) bacterial mechanisms of antimicrobial resistance, a clinical approach to gram-negative resistance by $\beta$ lactamases, and phases of opportunistic infections associated with allogeneic stem cell transplantation in recipients. Relevant algorithms, such as an approach to bacterial meningitis and febrile neutropenia, based on published infectious disease guidelines, are reproduced as well.

Many chapters have a segment near the end entitled Key Concepts, which reviews the chapter's relevant points. It would be advantageous to see this segment expanded in future editions so it could function as a quick reference for the reader searching for rapid answers. Another future consideration would be to provide clinical scenarios along with questions accompanying each chapter to reinforce the practical application of the information.

In summary, Critical Care Medicine: Principle of Diagnosis and Management in the Adult, 5th Edition is a mid-length textbook that lies somewhere between a review and a desk reference book, straddling this boundary well. It is an approachable, unintimidating resource with strengths that include a sophisticated consideration of the evidencebased literature and high-quality figures and images that seamlessly blend with the text. This book is ideally suited for both trainees preparing for critical care certification and seasoned health care professionals working in critical care environments. This textbook has happily found a permanent home on my library shelf.

Conflict of interest All authors declare that they have no conflict of interest.

Editorial responsibility This submission was handled by Dr. Steven Backman, Associate Editor, Canadian Journal of Anesthesia.

Publisher's Note Springer Nature remains neutral with regard to jurisdictional claims in published maps and institutional affiliations. 